\title{
Association between obstructive sleep apnea and thyroid cancer incidence: a national health insurance data study
}

\author{
Jae Hoon $\mathrm{Cho}^{1}$ (D) \\ Received: 17 August 2021 / Accepted: 17 August 2021 / Published online: 25 August 2021 \\ (c) The Author(s), under exclusive licence to Springer-Verlag GmbH Germany, part of Springer Nature 2021
}

\section{Dear Editor-in-Chief,}

I would like to respond to Lai et al.'s comment on "The Association Between Obstructive Sleep Apnea and Thyroid Cancer: A Study of National Health Insurance Data," recently published in this journal. First of all, thank you for pointing out the shortcomings of our study. I hope that this commentary and our responses to it will help readers better understand our paper.

Question 1) Why was the body mass index, an important factor in obstructive sleep apnea, not analyzed?

I agree that not taking body mass index into account in studies of patients with obstructive sleep apnea can be very problematic. This is because body mass index is a major factor influencing the severity of obstructive sleep apnea. The Korean National Health Insurance claim data used in this study did not include body mass index data. However, in Korea, free health check-ups are conducted every 2 years, and the records can be included in the claim data for analysis. The problem was that at the time of the analysis, only about $15 \%$ of the people who received the health checkup were, so the number of subjects was too small, and the power of analysis had to be reduced. Recently, $48 \%$ of the people are undergoing health checkups, and many studies are analyzing body mass index now.

Question 2) Why is person-year information missing?

This reply refers to the comment available online at https://doi.org/ 10.1007/s00405-021-07028-5.

Jae Hoon Cho

jaehoon@kuh.ac.kr

1 Department of Otorhinolaryngology-Head and Neck Surgery, College of Medicine, Konkuk University, 4-12 Hwayang-dong, Gwangjin-gu, Seoul 143-729, Korea
Although person-year is important information, it can be roughly estimated if you know the number of participants and the average follow-up period, and I thought that it was a number that had no meaning for general readers. However, I think that I should have provided person-year information for accurate judgment.

Question 3) Should all obstructive sleep apnea patients be screened for thyroid cancer, according to this paper?

Of course not. This paper is a retrospective observational study, and it did not analyze important variables such as body mass index or apnea-hypopnea index, and the mortality rate of thyroid cancer is very low. Considering all these factors, it is not a step to recommend aggressive screening. I think the role of this paper is to suggest the association between obstructive sleep apnea and thyroid cancer.

Question 4) The association between sleep apnea and thyroid cancer claimed in this study may have been distorted by several confounders.

I totally agree. I think it is probably a limitation of all observational studies. Therefore, this study alone cannot say that OSA causes thyroid cancer. However, I think it is a suggestion of such a possibility. To prove this, more detailed research is needed in the future, but I think there will be limitations because randomized control trial is not possible due to the nature of the study.

Publisher's Note Springer Nature remains neutral with regard to jurisdictional claims in published maps and institutional affiliations. 